\title{
Molecular Epidemiology and Whole-Genome Analysis of Bovine Foamy Virus in Japan
}

\author{
Hirohisa Mekata ${ }^{1, *(\mathbb{C})}$, Tomohiro Okagawa ${ }^{2}$, Satoru Konnai ${ }^{2,3}$ and Takayuki Miyazawa ${ }^{4}$ \\ 1 Center for Animal Disease Control, University of Miyazaki, Miyazaki 889-2192, Japan \\ 2 Department of Advanced Pharmaceutics, Faculty of Veterinary Medicine, Hokkaido University, \\ Sapporo 060-0818, Japan; okagawa@vetmed.hokudai.ac.jp (T.O.); konnai@vetmed.hokudai.ac.jp (S.K.) \\ 3 Department of Disease Control, Faculty of Veterinary Medicine, Hokkaido University, \\ Sapporo 060-0818, Japan \\ 4 Laboratory of Virus-Host Coevolution, Institute for Frontier Life and Medical Sciences, Kyoto University, \\ Kyoto 606-8507, Japan; takavet@infront.kyoto-u.ac.jp \\ * Correspondence: mekata@cc.miyazaki-u.ac.jp; Tel./Fax: +81-985-58-7881
}

Citation: Mekata, H.; Okagawa, T.; Konnai, S.; Miyazawa, T. Molecular Epidemiology and Whole-Genome Analysis of Bovine Foamy Virus in Japan. Viruses 2021, 13, 1017. https: / / doi.org/10.3390/ v13061017

Academic Editor:

Helle Bielefeldt-Ohmann

Received: 23 April 2021

Accepted: 26 May 2021

Published: 28 May 2021

Publisher's Note: MDPI stays neutral with regard to jurisdictional claims in published maps and institutional affiliations.

Copyright: (C) 2021 by the authors. Licensee MDPI, Basel, Switzerland. This article is an open access article distributed under the terms and conditions of the Creative Commons Attribution (CC BY) license (https:/ / creativecommons.org/licenses/by/ $4.0 /)$.

\begin{abstract}
Bovine foamy virus (BFV) is a member of the foamy virus family in cattle. Information on the epidemiology, transmission routes, and whole-genome sequences of BFV is still limited. To understand the characteristics of BFV, this study included a molecular survey in Japan and the determination of the whole-genome sequences of $30 \mathrm{BFV}$ isolates. A total of $30(3.4 \%, 30 / 884)$ cattle were infected with BFV according to PCR analysis. Cattle less than 48 months old were scarcely infected with this virus, and older animals had a significantly higher rate of infection. To reveal the possibility of vertical transmission, we additionally surveyed 77 pairs of dams and 3-month-old calves in a farm already confirmed to have BFV. We confirmed that one of the calves born from a dam with BFV was infected. Phylogenetic analyses revealed that a novel genotype was spread in Japan. In conclusion, the prevalence of BFV in Japan is relatively low and three genotypes, including a novel genotype, are spread in Japan.
\end{abstract}

Keywords: genotype; Japan; phylogeny; prevalence; spumavirus

\section{Introduction}

Bovine foamy virus (BFV), formerly called bovine syncytial virus, bovine spumavirus and bovine spumaretrovirus, is a member of the foamy virus (FV) sub-family that includes simian, prosimian, feline, and equine FVs [1]. FVs belong to the sub-family Spumaretrovirinae in the family Retroviridae, and BFV belongs to the genus Bovispumavirus. The BFV genome consists of approximately 12,000 nucleotides, including three structural genes ( $\mathrm{g} a \mathrm{~g}$, pol, env) common in retroviruses and two accessory genes (bel-1, bel-2) characteristic of FVs, which are flanked by two identical long terminal repeats (LTRs). The bel-1 gene encodes the Tas protein, which functions as a transcriptional activator [2]. The bel-2 gene may encode the Bel-2 protein, but such a protein has never been unambiguously detected in any FV analyzed so far [3]. Conversely, spliced mRNA from the early portion of the sequence of bel-1 and the entire sequence of bel-2 encodes the Bet protein [4]. Bet proteins of simian and feline FVs function as antagonists of antiretroviral proteins (APOBEC3) $[5,6]$. However, the function of the Bet protein of BFV is still obscure. BFV expresses microRNAs (miRNAs) in vitro and in vivo, and one of them is known to support virus replication $[7,8]$. BFV is considered a nonpathogenic retrovirus, as are other FVs $[9,10]$. The oral mucosa is the most active replication site of simian and feline FVs [11,12], and close contact is the essential factor of $\mathrm{BFV}$ transmission [13]. Thus, the major route of BFV transmission is considered to be licking or sneezing by BFV-infected cattle. Perinatal transmission through colostrum or milk has been proposed since virus isolation [14]. However, the importance and frequency of perinatal transmission under natural conditions are still controversial. 
Although the number of reports is limited, BFV is thought to be endemic throughout the world [14-16]. The positive rates have been shown to be markedly different between countries and BFV's prevalence has ranged from $7 \%$ to $42 \%$ since $2000[14,15]$. Recently, BFV was first isolated in Japan [17], and two epidemiological surveys were reported [18,19]. These studies reported that the virus was endemic in the Kanto, Hokkaido, and Kyushu areas of Japan, and the prevalence ranged from $12 \%$ to $16 \%$. The surveys were mostly conducted in the Kanto area, and one farm each was sampled in the Hokkaido, Tohoku, and Kyushu areas. Thus, an additional survey somewhere other than the Kanto area is required to understand BFV infection in Japan (Figure 1).

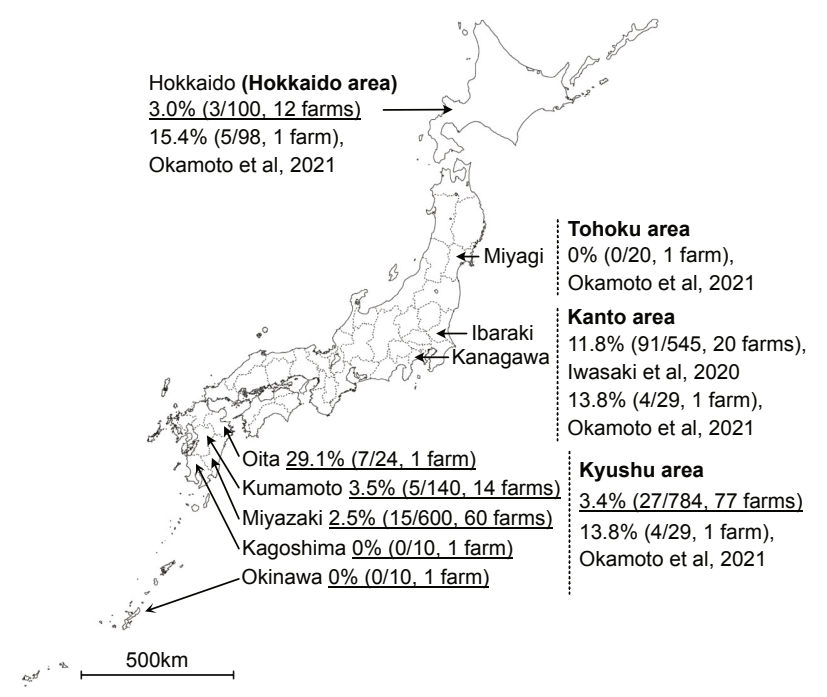

Figure 1. Map of Japan showing the positivity rate of bovine foamy virus (BFV) for each area. The map indicates the six prefectures (Hokkaido, Oita, Kumamoto, Miyazaki, Kagoshima and Okinawa) where sampling was performed in this study, and three prefectures (Miyagi, Ibaraki and Kanagawa) where sampling was performed in previous studies $[18,19]$. The BFV positivity rate is indicated by $\%$, and the parentheses show the numbers of positive and tested samples and the number of tested farms in each prefecture and area. The surveys conducted by this study are indicated with underlines.

FVs are thought to be ancient retroviruses and to have coevolved with host species [20,21]. Due to their nonpathogenic nature, broad tissue tropism, high packaging capacity and low genotoxicity, FVs are under development for novel viral vectors for gene delivery and vaccination [22-24]. Furthermore, a recent study suggested that FVs might have an antitumor effect through miRNA expression [25]. Thus, whole-genome analyses of FVs are important for developing viral vectors and for understanding evolutionary biology.

In this study, we analyzed the prevalence of BFV in the Kyushu and Hokkaido areas in Japan, the possibility of vertical transmission and the whole viral sequences of $30 \mathrm{BFV}$ isolates. This study will contribute to the understanding of the character and evolution of BFV.

\section{Materials and Methods}

\subsection{Samples}

Blood samples used for a molecular epidemiological survey were collected from a total of 884 cattle from 89 farms in the Hokkaido (12 farms: $n=100)$, Kumamoto (14 farms: $n=140$ ), Oita (1 farm: $n=24)$, Miyazaki (60 farms: $n=600)$, Kagoshima (1 farm: $n=10)$ and Okinawa ( 1 farm: $n=10$ ) prefectures in Japan from November 2016 to March 2020 by local clinical veterinarians. The number of samples per farm was 10 except for a farm in Oita $(n=24)$ and 4 farms $(n=6,6,5$, and 3$)$ in the Hokkaido prefecture. The samples were collected from cattle with a broad age range (0-48 months old: $n=255,48-84$ months old: $n=265,84-120$ months old: $n=221,>120$ months old: $n=110$, unknown: $n=33$ ) and mainly from Japanese Black cattle (Holstein: $n=116$, Japanese Black: $n=757$, cross-breed: 
$n=9$, others: $n=2$ ). Apart from the above, additional samples were collected from 77 Holstein pairs of dams and 3-month-old calves on a farm with confirmed BFV infection in June 2020 to assess the possibility of dam-to-calf transmission of BFV.

\subsection{DNA Extraction and Nested PCR}

Genomic DNA was extracted from whole blood using a Wizard Genomic DNA Purification Kit (Promega, Fitchburg, MA, USA) or the magLEAD system (Precision System Science, Chiba, Japan) according to the manufacturer's instructions. The DNA concentration was determined using a NanoDrop 8000 spectrophotometer (Thermo Fisher Scientific, Waltham, MA, USA), and samples were diluted to $20 \mathrm{ng} / \mu \mathrm{L}$. The extracted DNA was screened for the env gene of BFV by nested PCR, and the primer sets used were described in a previous report [17].

\subsection{Whole-Genome Amplification, Library Preparation and Next-Generation Sequencing}

The nucleotide diversity among the six available full-genome sequences that we retrieved from GenBank was calculated with windows of 200 bases and a 25-base step using DnaSP v6.12 [26]. Several primers were designed from the most conserved region and both ends of BFV by using Primer3Plus software (http:/ / www.bioinformatics.nl/ cgi-bin/primer3plus/primer3plus.cgi (accessed on 14 May 2020)). The designed primer pairs were tested for PCR amplification using BFV-positive genomes, and the best pairs (BFV1-6061: Forward 5'-TGTGGTGGAAAGACCACCCGGAAATAAGCAAGGGC-3', Reverse 5'-TGTCTTGTGTTGGGAGTGTTGTTCAGAGCAAG-3'; BFV5951-12001: Forward 5' AAGCGGCTCTTAACGAAACTGTTGGC-3' ${ }^{\prime}$, Reverse $5^{\prime}$-ATTGTTGTGACCTTCTCCAAT CTTTAGTGGATTC- $3^{\prime}$ ) were used for further studies. The amplification of two amplicons (BFV1-6052 and BFV5952-12001) covering whole viral genomes was performed in a reaction mixture containing $12.5 \mu \mathrm{L}$ of PrimeSTAR Max Premix (TaKaRa Bio, Kusatsu, Japan), a primer pair (BFV1-6052 or BFV5952-12001) for each amplicon at $0.3 \mu \mathrm{M}, 1 \mu \mathrm{L}$ of the extracted DNA and enough PCR-grade water to reach a final volume of $25 \mu \mathrm{L}$. Amplification was performed under the following conditions: an initial PCR activation step at $94{ }^{\circ} \mathrm{C}$ for $5 \mathrm{~s}, 5$ cycles of denaturation at $98^{\circ} \mathrm{C}$ for $10 \mathrm{~s}$ and annealing and extension at $70{ }^{\circ} \mathrm{C}$ for $6 \mathrm{~min}$, and 40 additional cycles with an annealing and extension temperature of $68^{\circ} \mathrm{C}$. Amplification of PCR products was confirmed by gel electrophoresis. The methods of purification of PCR products, library preparation and next-generation sequencing followed those in our previous report [27].

\subsection{Data Analysis of Next-Generation Sequencing}

The sequences generated by next-generation sequencing were analyzed using the CLC Genomics Workbench 11 software (Qiagen, Venlo, The Netherlands). The sequences were then processed to remove primers and low-quality sequences and mapped to reference genomes of the BFV strain NU (accession No. LC510607). The complete sequences of the BFVs that were determined in this study were submitted to the DDBJ under accession Nos. LC622406-35.

\subsection{Phylogenetic Analysis}

The BFV viral genome sequences that were obtained in this study and the available full-genome sequences that we retrieved from GenBank were aligned using ClustalW. Evolutionary distances were computed using Kimura's two-parameter model. Molecular phylogenetic trees were constructed using the neighbor-joining method with MEGA7 software [28]. A total of 1000 bootstrap replicates were used to derive the phylogenetic trees.

\subsection{Statistical Analysis}

The chi-square test was used to compare the positive number of infected farms and cattle for each prefecture, age, and cattle breed. These analyses were performed using 
GraphPad Prism 6 software (GraphPad Software, San Diego, CA, USA). $p<0.05$ was considered statistically significant in this study.

\section{Results}

In total, 12 of the $89(13.4 \%)$ farms and 30 of the $884(3.4 \%)$ cattle were positive for BFV according to nested PCR (Table 1). The number of positive farms and the positivity rate for each prefecture were as follows: Miyazaki $(n=8,13.3 \%)$, Kumamoto $(n=1,7.1 \%)$, Oita $(n=1,100 \%)$, Okinawa $(n=0,0 \%)$, Kagoshima $(n=0,0 \%)$ and Hokkaido $(n=2,16.6 \%)$. The number of positive cattle and the positivity rate for each prefecture were as follows: Miyazaki $(n=15,2.5 \%)$, Kumamoto $(n=5,3.5 \%)$, Oita $(n=7,29.1 \%)$, Okinawa $(n=0,0 \%)$, Kagoshima $(n=0,0 \%)$, and Hokkaido $(n=3,3.0 \%)$. The numbers of positive farms and cattle were compared among the Miyazaki, Kumamoto, and Hokkaido prefectures since only one farm was investigated in the Oita, Kagoshima, and Okinawa prefectures. No significant differences in the numbers of positive farms $(p=0.77)$ and cattle $(p=0.75)$ were confirmed among these prefectures. In this initial study, no PCR positivity was confirmed among cattle less than 48 months old $(n=255)$ (Table 2). Conversely, $4.9 \%(13 / 265), 4.3 \%$ $(9 / 221)$, and $6.7 \%(7 / 110)$ of the cattle were PCR-positive at $48-84,84-120$, and more than 120 months old, respectively. A significant difference among the age groups $(p=0.0029)$ was observed, as was a higher rate of BFV infection in older animals. No difference in the BFV positivity rate was observed between Holstein $(n=4,3.4 \%)$ and Japanese Black cattle $(n=25,3.3 \%)$.

Table 1. Prevalence of bovine foamy virus on 89 farms in the Hokkaido and Kyushu areas in Japan.

\begin{tabular}{cccc}
\hline Prefecture & Area & $\begin{array}{c}\text { No. of Positive Farms/Tests } \\
\text { (Positive Rate) }\end{array}$ & $\begin{array}{c}\text { No. of Positive Cattle/Tests } \\
\text { (Positive Rate) }\end{array}$ \\
\hline Miyazaki & Kyushu & $8 / 60(13.3 \%)$ & $15 / 600(2.5 \%)$ \\
Kumamoto & Kyushu & $1 / 14(7.1 \%)$ & $5 / 140(3.5 \%)$ \\
Oita & Kyushu & $1 / 1(100 \%)$ & $7 / 24(29.1 \%)$ \\
Okinawa & Kyushu & $0 / 1(0 \%)$ & $0 / 10(0 \%)$ \\
Kagoshima & Kyushu & $0 / 1(0 \%)$ & $0 / 10(0 \%)$ \\
Hokkaido & Hokkaido & $2 / 12(16.6 \%)$ & $3 / 100(3.0 \%)$ \\
\hline Total & & $12 / 89(13.4 \%)$ & $30 / 884(3.4 \%)$ \\
\hline
\end{tabular}

Table 2. Prevalence of bovine foamy virus among the age groups of cattle.

\begin{tabular}{ccc}
\hline Age (Months) & No. of Samples & No. of Positive Cattle (\%) \\
\hline $0-48$ & 255 & $0(0)$ \\
$48-84$ & 265 & $13(4.9)$ \\
$84-120$ & 221 & $9(4.3)$ \\
$>120$ & 110 & $7(6.7)$ \\
Unknown & 33 & $1(3.0)$ \\
\hline Total & 884 & $30(3.4)$ \\
\hline
\end{tabular}

Previous studies reported that BFV was isolated from milk and uterine cells $[14,29]$. Although cattle less than 48 months old were scarcely confirmed to have BFV infection in this study, the virus was thought to have potential for dam-to-calf transmission. To investigate this possibility, we performed an additional survey in 77 pairs of dams and 3-month-old calves on a farm confirmed to have BFV infection. One of every seven calves born from a dam with BFV was infected with BFV. Thus, the possibility of dam-to-calf transmission of BFV was confirmed. BFVs were successfully isolated from three blood samples of seven PCR-positive dams. The three virus isolates were named BFV11996, BFV13294, and BFV13630 and were used for further experiments.

To design primers for amplifying the whole genome of BFV, we performed sliding window analysis among the six isolates of BFV with deposited whole-genome sequences in 
GenBank (accession Nos. AY134750, LC510606-7, NC001831, and JX307861-2) (Figure 2a). The sequences of the pol gene corresponding to positions 5887-6086 of the BFV Riems strain (accession No. JX307862) were the most conserved region among the six BFVs. Thus, we designed primers from this conserved region and amplified 32 pairs of BFV amplicons (Figure 2b). Finally, we obtained 30 complete consensus sequences for BFVs identified in the Miyazaki, Kumamoto, Oita, and Hokkaido prefectures in Japan.
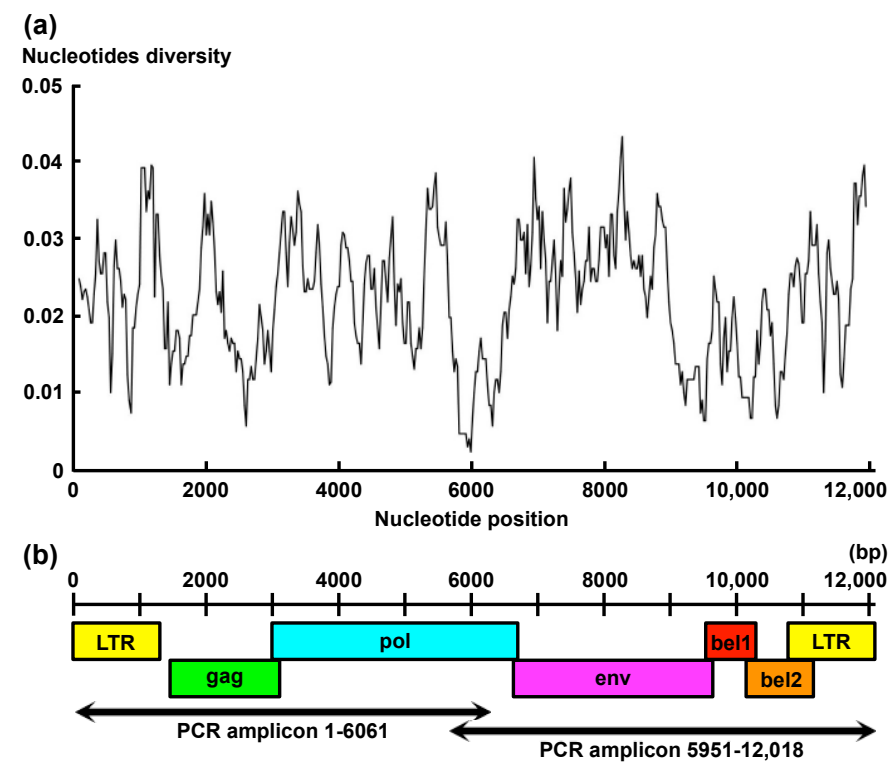

(c)

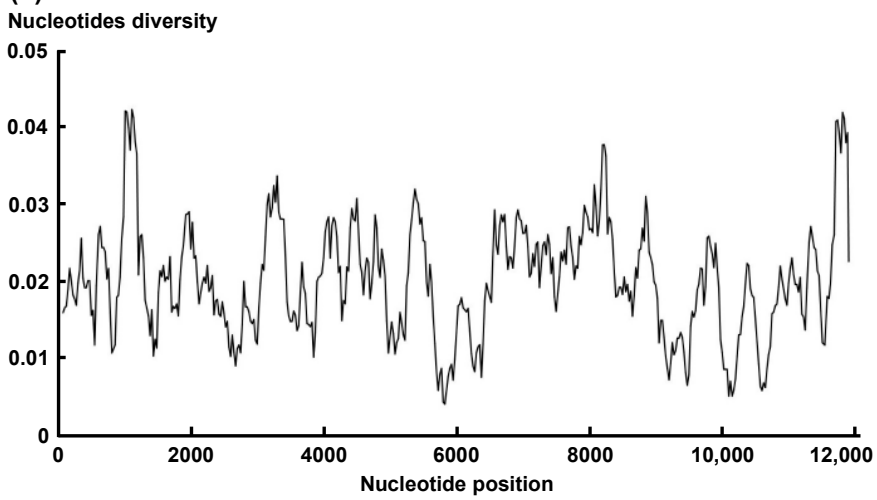

Figure 2. Genome structure and nucleotide diversity of bovine foamy virus (BFV). The nucleotide diversity among the six full-genome sequences of BFVs deposited in GenBank (accession Nos. AY134750, LC510606-7, NC001831, and JX307861-2) is presented. The nucleotide diversity was calculated using DnaSP v6.12 [26]. A sliding window method with windows of 200 nucleotides and steps of 25 nucleotides was used to calculate the nucleotide diversity across the entire alignment (a). Genome structure and locations of PCR amplicons for whole-genome analysis are presented. The BFV genome consists of approximately 12,000 nucleotides, including three structural genes (gag, pol, env) and two accessory genes (bel-1, bel-2) flanked by two identical long terminal repeats (LTRs). The locations of the two PCR amplicons for whole-genome analysis are shown (b). The nucleotide diversity of full-genome sequences among the $36 \mathrm{BFV}$ s that were obtained in this study and deposited in GenBank is presented (c).

The phylogenetic analysis of the whole viral sequence indicated that there were three genotypes among the BFVs (Figure 3). Most isolates in this study were of the Asia/USA genotype. Three isolates from Miyazaki and Hokkaido were of the European genotype. Furthermore, a novel genotype, named "Third genotype", was identified, and four isolates from Oita were in this group. The nucleotide distances between isolates in the Europe and Third, Europe and Asia/USA, and Third and Asia/USA genotypes were in the ranges 
$2.9-3.3 \%, 3.6-4.2 \%$, and $3.8-4.2 \%$, respectively. The nucleotide similarity rates of LTR and gag, pol, env, bel-1 and bel-2 genes among all isolates were $88.6 \%, 91.1 \%, 90.6 \%, 89.6 \%$, $92.4 \%$, and $92.8 \%$, respectively. The deletion of 12 nucleotides (amino acid position of Gag protein: 170-173) was confirmed in three isolates of BFV (BFV/Kumamoto/A-6/2019, $\mathrm{BFV} /$ Kumamoto/A-7/2019, and BFV/Miyazaki/J-5/2018). A nucleotide substitution of a potential initiator codon in the bel-2 gene (corresponding to position 10,122 of the BSV Riems strain), resulting in protein substitution from methionine to isoleucine, was confirmed in three isolates of BFV of the European genotype (BFV/Miyazaki/I-9/2018, BFV/Miyazaki/D-9/2020, and BFV/Hokkaido/L-72/2016).

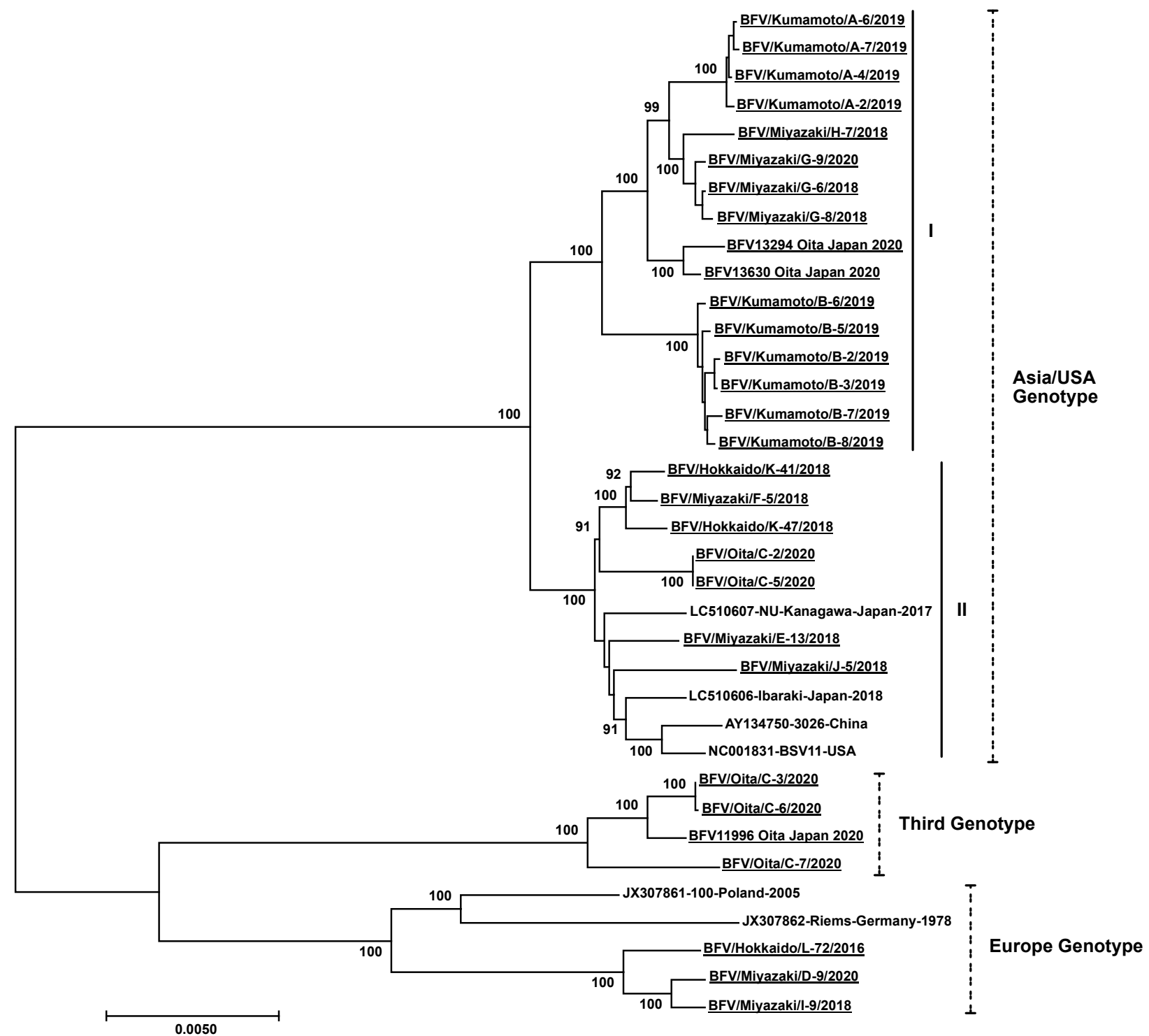

Figure 3. Phylogenetic analysis of the whole-genome sequences of bovine foamy virus (BFV). The analysis involved the whole-genome sequences of the $36 \mathrm{BFV}$ isolates at the nucleotide level. The names of the 27 isolates for which the whole viral sequence was identified in this study are denoted as "BFV", "isolation prefecture", "name of isolate", and "isolation year" separated by slashes and with underlines (e.g., BFV/Miyazaki/G-6/2018). Three isolates of the virus isolated in this study are named as "name of isolate", "isolation prefecture", "Japan", and "isolation year" with underlines (e.g., BFV13294 Oita Japan 2020). The sequences retrieved from GenBank are denoted as "accession No.", "name of strain", "isolation (prefecture) country", and "isolation year" separated by hyphens (e.g., LC150607-NU-Kanagawa-Japan-2017). Bootstrap values of more than $80 \%$ (1000 replicates) are shown next to the branches. The scale bar indicates the number of substitutions per site. The vertical broken and full bars show the classification of genotypes (Asia/USA, Third, and Europe) and clusters (I, II) of BFV, respectively. 
Finally, we performed sliding window analysis among the 36 full-genome sequences of $\mathrm{BFV}$ (Figure 2c). The most variable region was confirmed in the LTR region corresponding to positions 1037-1236 of the BFV Riems strain. Conversely, the most conserved region was confirmed in the pol region corresponding to positions 5749-5948 of the BFV Riems strain.

\section{Discussion}

This is a report of a molecular epidemiological study of BFV in the Kyushu and Hokkaido areas in Japan. We revealed that $3.4 \%$ of cattle are infected with BFV. The whole viral sequences of 30 isolates were determined, and three genotypes, including a novel genotype, were confirmed to be endemic in Japan.

To reveal the prevalence of BFV in Japan, we investigated 784 samples from 77 farms in the Kyushu area (Miyazaki, Kagoshima, Kumamoto, Oita, and Okinawa) and 100 samples from 12 farms in the Hokkaido area (Table 1 and Figure 1). The positivity rates were similar in the Kyushu $(3.4 \% ; 27 / 784)$ and Hokkaido $(3.0 \% ; 3 / 100)$ areas. A previous study reported that $16.7 \%$ of cattle $(91 / 545,20$ farms) in the Kanto area in Japan were infected with BFV [18]. This previous report included an increased number of collected samples if BFV-positive cattle were detected on a farm. Thus, the prevalence of BFV might have been slightly overestimated. Nevertheless, the rate of BFV infection could be different among areas in Japan due to the difference in stocking density, the average number of feeding years and the frequency of movement among farms. The prevalence rates of BFV in Germany $(7 \%)$ and Poland $(30-42 \%)$ were previously reported $[14,15]$. All the surveys conducted in Japan used PCR methods, and Iwasaki et al. confirmed that positive results were coincident when using agar gel immunodiffusion (AGID) and PCR tests [18]. The surveys conducted in foreign countries used a variety of serological methods, mainly ELISA tests. Generally, ELISA has a higher sensitivity than AGID tests. Thus, the epidemiological surveys conducted in foreign countries might have higher sensitivity than those conducted in Japan.

A significant difference was found between the age of cattle and BFV positivity in this study. A similar tendency was reported in previous studies $[16,19]$. This tendency suggests that BFV is mainly transmitted through the horizontal route under certain conditions. In contrast, some studies conducted in other countries reported no difference between the age of cattle and BFV positivity [14]. This result might be affected by the breeding style of each country, especially the duration of the feeding period with dams and calves. Simian and feline FVs are released from the oral mucosa and thought to be transmitted through biting $[11,12,30]$. However, cows are milder animals and rarely attack by biting. A previous study confirmed that spraying the infectious agent into the throat was the most successful route of BFV infection [13]. Thus, BFV might be horizontally transmitted by licking and sneezing. Cattle less than 48 months old were scarcely PCR-positive in this study. On the other hand, BFV is thought to have the potential for vertical transmission due to virus isolation from milk and the placenta and uterus [14,29]. Thus, BFV infection of 77 pairs of dams and calves from a farm with BFV infection was confirmed. We used 3-month-old calves because vertical transmission of Theileria orientalis was not detectable in 1-month-old calves, but was detectable in 3-month-old calves by PCR [31]. One dam and calf pair was infected with BFV. Thus, BFV might be transmitted via a vertical route, but the frequency is considered to be very low considering the prevalence among young cattle.

BFV was divided into two genotypes (Europe and Asia/USA) in the past [19,32]. In addition, this study identified a novel genotype, named the Third genotype (Figure 3). The phylogenetic analyses based on the sequences of all the coding and noncoding regions of BFV also classified all the isolates into identical genotypes based on the whole viral sequence (Figure 4). Thus, the Third genotype is thought to be completely branched and established in BFV. Several serotypes have been reported in simian and and two in feline FV $[33,34]$. The nucleotide distance between the two serotypes of feline FV was approximately $7 \%$. The nucleotide distances among the three genotypes of BFV was in the range $2.9-4.2 \%$. Thus, we assume that there is no serological difference among the BFV 
genotypes. However, further studies are required. Although we identified four isolates of BFV clustered into the Third genotype, all isolates were identified on the same farm in Oita prefecture. Thus, we could not conclude that this genotype was spread throughout Japan. The Asia/USA genotype can be divided into two clusters based on whole viral sequences (Figure 3). The phylogenetic trees based on the sequences of the LTR and gag, pol, and env genes can also be divided into two clusters (Figure 4a-d). However, the phylogenetic trees based on the sequences of the bel-1 and bel-2 genes could not be divided because these genes were the most conserved regions (Figure 4e,f). Thus, branching of the two clusters might have occurred in recent decades and may be currently in progress. A similar tendency was observed in the phylogenetic analysis of bovine coronavirus [35]. Many Holstein cattle were imported from North America to Japan more than 20 years ago. However, few live cattle have been imported into Japan, except for importation which has occured from Australia in the last two decades due to the problems of transboundary diseases, such as foot-and-mouse disease and bovine spongiform encephalopathy. This policy might affect the branching of the Asia/USA genotype in Japan.

(a) LTR

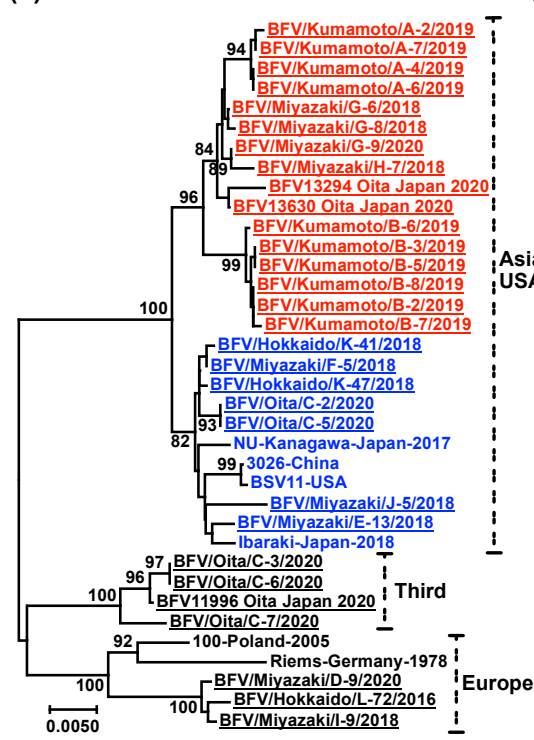

(b) gag

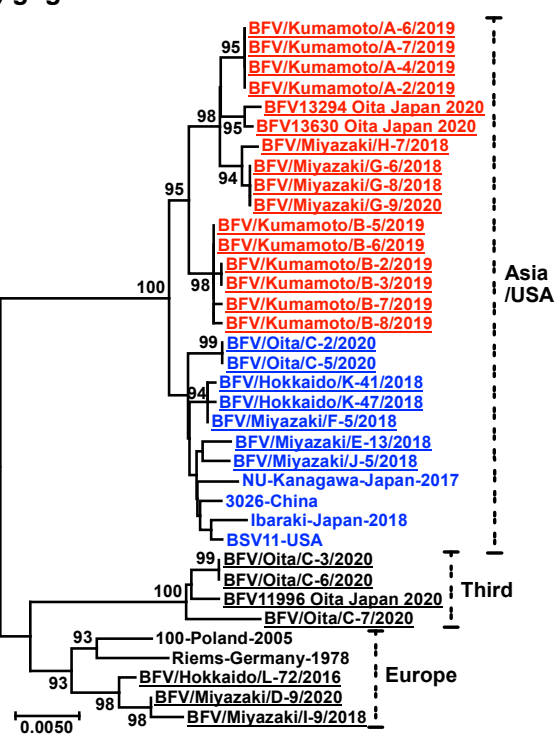

(e) bel-1

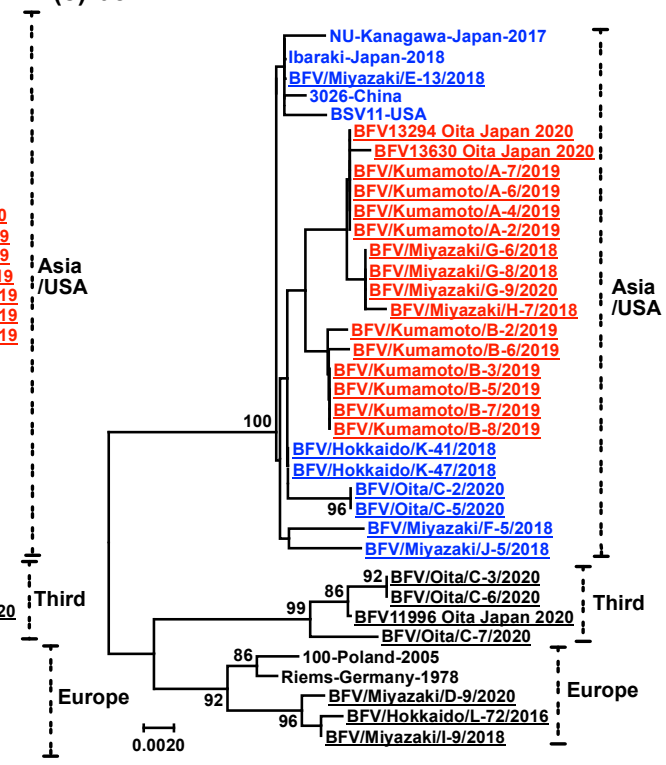

(c) pol

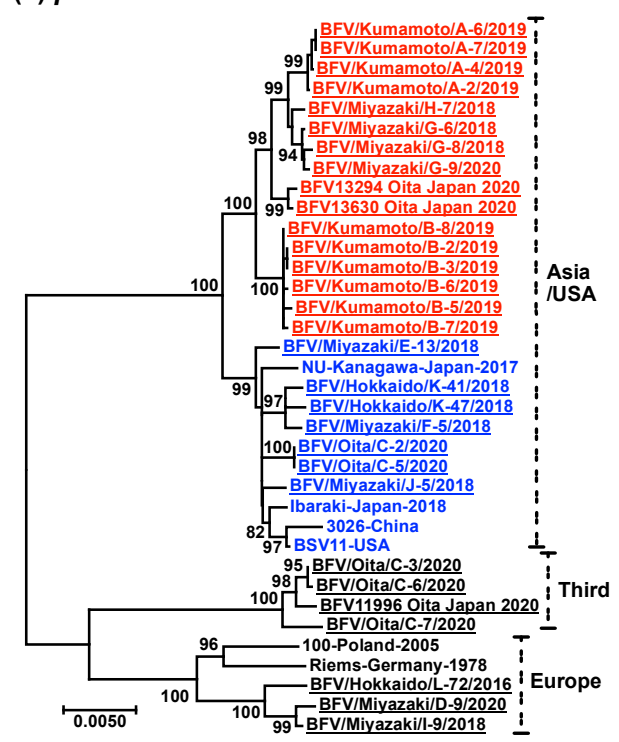

(f) bel-2

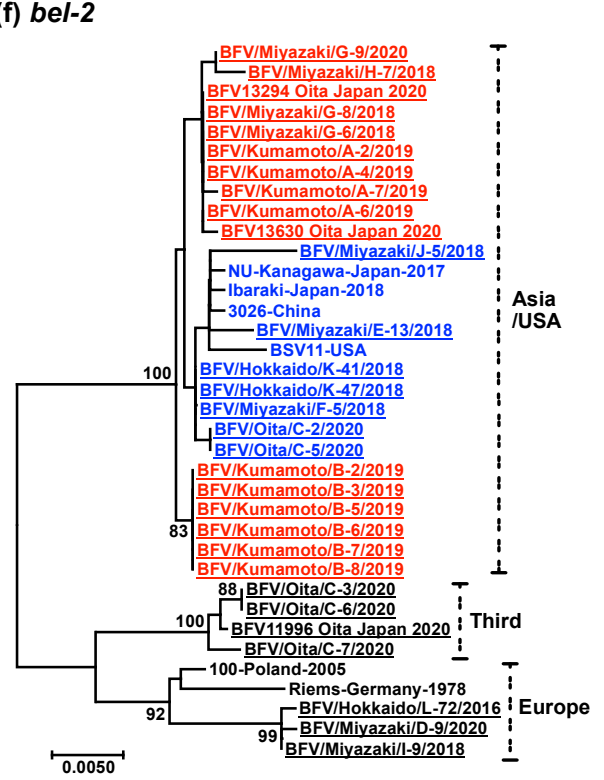

(d) env

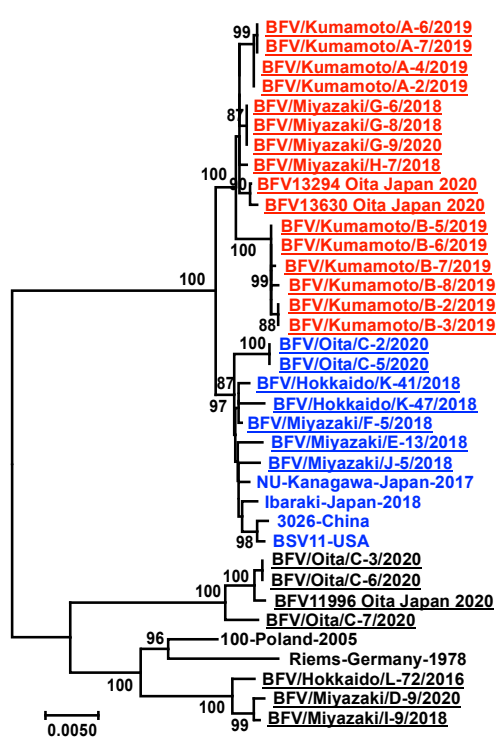

Figure 4. Phylogenetic analyses of the entire coding and noncoding genes of bovine foamy virus (BFV). Phylogenetic trees based on the entire LTR (a) and $g a g(\mathbf{b})$, pol (c), env (d), bel-1 (e), and bel-2 (f) genes. The 27 isolates for which the whole viral 
sequence was identified in this study are denoted as "BFV", "isolation prefecture", "name of isolate", and "isolation year" separated by slashes and with underlines (e.g., BFV/Miyazaki/G-6/2018). Three isolates of the virus isolated in this study are denoted as "name of isolate", "isolation prefecture", "Japan", and "isolation year" with underlines (e.g., BFV13294 Oita Japan 2020). The sequences retrieved from GenBank are denoted as "name of isolate", "isolation (prefecture) country", and "isolation year" with hyphens (e.g., NU-Kanagawa-Japan-2017). Bootstrap values of more than 80\% (1000 replicates) are shown next to the branches. The scale bars indicate the number of substitutions per site. The vertical broken bars show the classification of genotypes (Asia/USA, Third, and Europe). The clusters (I, II) of BFV within the Asia/USA genotype are colored with red and blue font.

The deletion of four amino acids in the Gag protein (170-173) was confirmed in three isolates of BFV. Amino acid exchange in the BFV Gag protein was reported to correlate with efficient amplification and cell-free transmission of BFV in vitro [36,37]. Although the deletion of four amino acids was not observed in previous studies, it might affect the amplification and transmission of BFV. The nucleotide similarities of $e n v(89.6 \%)$ and bel-2 $(92.8 \%)$ were the lowest and highest among BFVs. Although bel-2 was the most conserved gene, protein substitution of a potential initiator codon of Bel-2 protein was confirmed among three isolates of the European genotype identified in Japan. Bel-2 protein has not been unambiguously identified in simian, feline and bovine FVs. Thus, this observation supports the idea that the bel-2 protein does not exist in FVs. Conversely, the Bet protein is encoded by a spliced mRNA from the first part of the sequence of bel-1 and the entire sequence of bel-2 [4]. The amino substituted acid from methionine to isoleucine was located downstream of the splice acceptor [38]. Thus, the mutation of the potential initiator codon in the bel-2 gene would not affect Bet protein expression.

\section{Conclusions}

We found that the prevalence of BFV in Japan is 3.4\%, and three genotypes (Asia/USA, Europe, and Third), including a novel genotype (Third), are spread in Japan. To date, there is not enough information available regarding this virus throughout the world. Genetic information about BFV is important for the understanding viral evolution and the possibility of its effective utilization as a novel vector. Therefore, further studies are required to identify the importance and evolution of this virus.

Author Contributions: H.M. and T.M. designed and organized the study. H.M. and S.K. contributed to the blood sampling from cattle. H.M., T.O., and S.K. performed the laboratory assay. H.M. analyzed the data and wrote the manuscript. All authors have read and agreed to the published version of the manuscript.

Funding: This work was supported by the grants JSPS KAKENHI grant number $20 \mathrm{H} 03150$ and JST SATREPS grant number JPMJSA1908.

Institutional Review Board Statement: The blood samples were taken by clinical veterinarians based on the ethical guidelines of the belonging company or group. The survey was performed under the owner's consent. No further permissions by an Institutional Review Board were necessary.

Informed Consent Statement: Not applicable.

Data Availability Statement: The data that support the findings of this study are available from the corresponding author, H.M., upon reasonable request. The whole viral sequences of BFV obtained in this study are deposited in DDBJ (accession Nos. LC622406-35) and available at https:/ / www.ddbj. nig.ac.jp/index-e.html (accessed on 28 May 2021).

Acknowledgments: We kindly thank the many clinical veterinarians and owners for the collection of bovine blood samples.

Conflicts of Interest: The authors declare no conflict of interest. None of the funding sources influenced the design or performance of the experimental study, interpretation of the results or writing of the manuscript. 


\section{References}

1. Khan, A.S.; Bodem, J.; Buseyne, F.; Gessain, A.; Johnson, W.; Kuhn, J.H.; Kuzmak, J.; Lindemann, D.; Linial, M.L.; Löchelt, M.; et al. Spumaretroviruses: Updated taxonomy and nomenclature. Virology 2018, 516, 158-164. [CrossRef]

2. Tan, J.; Qiao, W.; Xu, F.; Han, H.; Chen, Q.; Geng, Y. Dimerization of BTas is required for the transactivational activity of bovine foamy virus. Virology 2008, 376, 236-241. [CrossRef]

3. Saïb, A.; de Thé, H. Molecular biology of the human foamy virus. J. Acquir. Immune Defic. Syndr. Hum. Retrovirol. 1996, 13, S254-S260. [CrossRef]

4. Materniak-Kornas, M.; Tan, J.; Heit-Mondrzyk, A.; Hotz-Wagenblatt, A.; Löchelt, M. Bovine foamy virus: Shared and unique molecular features in vitro and in vivo. Viruses 2019, 11, 1084. [CrossRef]

5. Löchelt, M.; Romen, F.; Bastone, P.; Muckenfuss, H.; Kirchner, N.; Kim, Y.B.; Truyen, U.; Rösler, U.; Battenberg, M.; Saib, A.; et al. The antiretroviral activity of APOBEC3 is inhibited by the foamy virus accessory Bet protein. Proc. Natl. Acad. Sci. USA 2005, 102, 7982-7987. [CrossRef] [PubMed]

6. Russell, R.A.; Wiegand, H.L.; Moore, M.D.; Schäfer, A.; McClure, M.O.; Cullen, B.R. Foamy virus Bet proteins function as novel inhibitors of the APOBEC3 family of innate antiretroviral defense factors. J. Virol. 2005, 79, 8724-8731. [CrossRef] [PubMed]

7. Cao, W.; Stricker, E.; Hotz-Wagenblatt, A.; Heit-Mondrzyk, A.; Pougialis, G.; Hugo, A.; Kuźmak, J.; Materniak-Kornas, M.; Löchelt, M. Functional analyses of bovine foamy virus-encoded miRNAs reveal the importance of a defined miRNA for virus replication and host-virus interaction. Viruses 2020, 12, 1250. [CrossRef] [PubMed]

8. Whisnant, A.W.; Kehl, T.; Bao, Q.; Materniak, M.; Kuzmak, J.; Löchelt, M.; Cullen, B.R. Identification of novel, highly expressed retroviral microRNAs in cells infected by bovine foamy virus. J. Virol. 2014, 88, 4679-4686. [CrossRef] [PubMed]

9. Falcone, V.; Schweizer, M.; Neumann-Haefelin, D. Replication of primate foamy viruses in natural and experimental hosts. Curr. Top. Microbiol. Immunol. 2003, 277, 161-180. [CrossRef]

10. Meiering, C.D.; Linial, M.L. Historical perspective of foamy virus epidemiology and infection. Clin. Microbiol. Rev. 2001, 14, 165-176. [CrossRef]

11. Alke, A.; Schwantes, A.; Zemba, M.; Flügel, R.M.; Löchelt, M. Characterization of the humoral immune response and virus replication in cats experimentally infected with feline foamy virus. Virology 2000, 275, 170-176. [CrossRef] [PubMed]

12. Falcone, V.; Leupold, J.; Clotten, J.; Urbanyi, E.; Herchenröder, O.; Spatz, W.; Volk, B.; Böhm, N.; Toniolo, A.; NeumannHaefelin, D.; et al. Sites of simian foamy virus persistence in naturally infected African green monkeys: Latent provirus is ubiquitous, whereas viral replication is restricted to the oral mucosa. Virology 1999, 257, 7-14. [CrossRef] [PubMed]

13. Johnson, R.H.; de la Rosa, J.; Abher, I.; Kertayadnya, I.G.; Entwistle, K.W.; Fordyce, G.; Holroyd, R.G. Epidemiological studies of bovine spumavirus. Vet. Microbiol. 1988, 16, 25-33. [CrossRef]

14. Romen, F.; Backes, P.; Materniak, M.; Sting, R.; Vahlenkamp, T.W.; Riebe, R.; Pawlita, M.; Kuzmak, J.; Löchelt, M. Serological detection systems for identification of cows shedding bovine foamy virus via milk. Virology 2007, 364, 123-131. [CrossRef]

15. Materniak-Kornas, M.; Osiński, Z.; Rudzki, M.; Kuźmak, J. Development of a recombinant protein-based ELISA for detection of antibodies against bovine foamy virus. J. Vet. Res. 2017, 61, 247-252. [CrossRef]

16. Jacobs, R.M.; Pollari, F.L.; McNab, W.B.; Jefferson, B. A serological survey of bovine syncytial virus in Ontario: Associations with bovine leukemia and immunodeficiency-like viruses, production records, and management practices. Can. J. Vet. Res. 1995, 59, 271-278. [PubMed]

17. Hachiya, Y.; Kimura, K.; Oguma, K.; Ono, M.; Horikita, T.; Sentsui, H. Isolation of bovine foamy virus in Japan. J. Vet. Med. Sci. 2018, 80, 1604-1609. [CrossRef]

18. Iwasaki, R.; Nakagiri, Y.; Yaguchi, Y.; Oguma, K.; Ono, M.; Horikita, T.; Sentsui, H. Survey of bovine foamy virus infection among cattle in Japan and comparison with bovine leukemia virus infection. J. Vet. Med. Sci. 2020, 82, 615-618. [CrossRef]

19. Okamoto, M.; Oguma, K.; Yamashita-Kawanishi, N.; Ichijo, T.; Hatama, S.; Endo, M.; Ishikawa, M.; Haga, T. Genomic characterization and distribution of bovine foamy virus in Japan. J. Vet. Med. Sci. 2020, 82, 1607-1613. [CrossRef] [PubMed]

20. Switzer, W.M.; Salemi, M.; Shanmugam, V.; Gao, F.; Cong, M.E.; Kuiken, C.; Bhullar, V.; Beer, B.E.; Vallet, D.; Gautier-Hion, A.; et al. Ancient co-speciation of simian foamy viruses and primates. Nature 2005, 434, 376-380. [CrossRef] [PubMed]

21. Katzourakis, A.; Gifford, R.J.; Tristem, M.; Gilbert, M.T.P.; Pybus, O.G. Macroevolution of complex retroviruses. Science 2009, 325, 1512. [CrossRef] [PubMed]

22. Goodman, M.A.; Arumugam, P.; Pillis, D.M.; Loberg, A.; Nasimuzzaman, M.; Lynn, D.; van der Loo, J.C.M.; Dexheimer, P.J.; Keddache, M.; Bauer, T.R., Jr.; et al. Foamy virus vector carries a strong insulator in its long terminal repeat which reduces its genotoxic potential. J. Virol. 2018, 92, e01639-17. [CrossRef] [PubMed]

23. Sweeney, N.P.; Meng, J.; Patterson, H.; Morgan, J.E.; McClure, M. Delivery of large transgene cassettes by foamy virus vector. Sci. Rep. 2017, 7, 8085. [CrossRef] [PubMed]

24. Ledesma-Feliciano, C.; Hagen, S.; Troyer, R.; Zheng, X.; Musselman, E.; Lukic, D.S.; Franke, A.M.; Maeda, D.; Zielonka, J.; Münk, C.; et al. Replacement of feline foamy virus bet by feline immunodeficiency virus vif yields replicative virus with novel vaccine candidate potential. Retrovirology 2018, 15, 38. [CrossRef] [PubMed]

25. Hashimoto-Gotoh, A.; Kitao, K.; Miyazawa, T. Persistent infection of simian foamy virus derived from the Japanese macaque leads to the high-level expression of microRNA that resembles the miR-1 microRNA precursor family. Microbes Environ. 2020, 35, ME19130. [CrossRef] [PubMed] 
26. Rozas, J.; Ferrer-Mata, A.; Sánchez-DelBarrio, J.C.; Guirao-Rico, S.; Librado, P.; Ramos-Onsins, S.E.; Sánchez-Gracia, A. DnaSP 6: DNA sequence polymorphism analysis of large data sets. Mol. Biol. Evol. 2017, 34, 3299-3302. [CrossRef]

27. Sato, Y.; Mekata, H.; Sudaryatma, P.E.; Kirino, Y.; Yamamoto, S.; Ando, S.; Sugimoto, T.; Okabayashi, T. Isolation of severe fever with thrombocytopenia syndrome virus from various tick species in area with human severe fever with thrombocytopenia syndrome cases. Vector Borne Zoonotic Dis. 2021, 5, 378-384. [CrossRef]

28. Kumar, S.; Stecher, G.; Tamura, K. MEGA7: Molecular evolutionary genetics analysis version 7.0 for bigger datasets. Mol. Biol. Evol. 2016, 33, 1870-1874. [CrossRef]

29. Ruckerbauer, G.M.; Sugden, E.A.; Bouillant, A.M. A comparison of the bovine leukemia and bovine syncytial virus status in utero-tubal cells recovered from fluids used to flush the uterus and oviducts of BLV-infected, superovulated cattle. Ann. Rech. Vet. 1988, 19, 19-26.

30. Schweizer, M.; Falcone, V.; Gänge, J.; Turek, R.; Neumann-Haefelin, D. Simian foamy virus isolated from an accidentally infected human individual. J. Virol. 1997, 71, 4821-4824. [CrossRef]

31. Mekata, H.; Minamino, T.; Mikurino, Y.; Yamamoto, M.; Yoshida, A.; Nonaka, N.; Horii, Y. Evaluation of the natural vertical transmission of Theileria orientalis. Vet. Parasitol. 2018, 263, 1-4. [CrossRef]

32. Hechler, T.; Materniak, M.; Kehl, T.; Kuzmak, J.; Löchelt, M. Complete genome sequences of two novel European clade bovine foamy viruses from Germany and Poland. J. Virol. 2012, 86, 10905-10906. [CrossRef] [PubMed]

33. Johnston, P.B. Taxonomic features of seven serotypes of simian and ape foamy viruses. Infect. Immun. 1971, 3, 793-799. [CrossRef]

34. Winkler, I.G.; Flügel, R.M.; Löchelt, M.; Flower, R.L.P. Detection and molecular characterization of feline foamy virus serotypes in naturally infected cats. Virology 1998, 247, 144-151. [CrossRef] [PubMed]

35. Mekata, H.; Hamabe, S.; Sudaryatma, P.E.; Kobayashi, I.; Kanno, T.; Okabayashi, T. Molecular epidemiological survey and phylogenetic analysis of bovine respiratory coronavirus in Japan from 2016 to 2018. J. Vet. Med. Sci. 2020, 82, 726-730. [CrossRef] [PubMed]

36. Bao, Q.; Hipp, M.; Hugo, A.; Lei, J.; Liu, Y.; Kehl, T.; Hechler, T.; Löchelt, M. In vitro evolution of bovine foamy virus variants with enhanced cell-free virus titers and transmission. Viruses 2015, 7, 5855-5874. [CrossRef] [PubMed]

37. Bao, Q.; Hotz-Wagenblatt, A.; Betts, M.J.; Hipp, M.; Hugo, A.; Pougialis, G.; Lei-Rossmann, J.; Löchelt, M. Shared and cell type-specific adaptation strategies of Gag and Env yield high titer bovine foamy virus variants. Infect. Genetics. Evol. 2020, 82, 104287. [CrossRef]

38. Bing, T.; Wu, K.; Cui, X.; Shao, P.; Zhang, Q.; Bai, X.; Tan, J.; Qiao, W. Identification and functional characterization of Bet protein as a negative regulator of BFV3026 replication. Virus Genes 2014, 48, 464-473. [CrossRef] 